\title{
Etiology and Outcomes in Elderly Patients Admitted For Acute Infections
}

\author{
Vinita Patil ${ }^{1}$, Elizabeth James ${ }^{2}$, Sangeeta Pednekar ${ }^{3}$ \\ ${ }^{1}$ Senior Resident, Department of Medicine, Lokmanya Tilak Municipal Medical College (LTMMC) and General Hospital, Sion, Mumbai, India, ${ }^{2}$ Assistant \\ Professor Department of Medicine, Believers Church Medical College, Thiruvalla, Kerala, ${ }^{3}$ Professor, Department of Medicine, Lokmanya Tilak Municipal \\ Medical College (LTMMC) and General Hospital, Sion, Mumbai, India.
}

\section{Abstract}

Background: Infectious diseases are a major cause of mortality in elderly. The present study aimed to assess various acute infections in elderly population admitting in our department and factors which are associated with their clinical outcomes. Subjects and Methods: Hundred patients, aged more than 65 years of age, having fever of less than 7 days' duration or having symptoms of any of the major system admitted in our department were included. Detailed history, clinical and laboratory work-up was done to diagnose the cause of infection. Mortality of the patients during hospital stay was noted and factors associated with it were ascertained from statistical analysis. Results: Mean age of the patients was 75.84 years, two-thirds being males. Hypertension and diabetes mellitus were the most common co morbidities. Pulmonary infections accounted for majority of the infections (43\%), urinary tract infections were the second most common cause of acute infection. Gastrointestinal infections (10\%), central nervous system infections (6\%), skin infections (4\%) were other less common causes. During hospitalization, the most common complication was respiratory failure. Overall, 15 patients died during hospital stay. We found higher APACHE-II score and presence of pulmonary infections to be significantly associated with in-hospital mortality. Conclusion: A multidisciplinary management of elderly patients with infections is required.

Keywords: Aged; Critical Care; ICU; Mortality; Sepsis.

Corresponding Author: Dr. Elizabeth James, Assistant Professor Department of Medicine, Believers Church Medical College, Thiruvalla, Kerala.

Received: December 2019

Accepted: December 2019

\section{Introduction}

Aging is associated with numerous physiological changes and progressive decline in body's ability to maintain physiological homeostasis. This results in alterations in organ functions, functional decline, multimorbidity, and frailty. ${ }^{[1]}$ Immunosenescence is the science how aging dependent changes in the immune system happen and how this may affect the elderly's ability to overcome external stressors. Immunosenescence is present in all older adults in varying degrees and there is a link between the degree of frailty and immunocompetency. ${ }^{[1]}$ As the immune system ages and the normal capabilities of defence against infections, malignant or autoreactive cells declines, increased susceptibility to infections, malignancy, autoimmune disorders, and impaired wound repair follow. Many older adults have mild degrees of immunosuppression as a result of immunosenescence, together with, age related organ changes, comorbidities, geriatric syndromes, frailty, malnutrition, functional dysfunction and, polypharmacy, all of which affect the prognosis of geriatric patients with infectious diseases.

Despite advances in antimicrobial therapy, infectious diseases continue to be a major cause of mortality in older adults. The diagnostic and therapeutic nuances of managing infections in older adults create special challenges for physicians. At the tertiary care level, which comprises of super specialty and medical college hospitals, there needs to be provision of separate indoor and outpatient clinics for elderly patients. The present study aimed to assess various acute infections in elderly population admitting in our department and factors which are associated with their clinical outcomes.

\section{Subjects and Methods}

\section{Study Design and Sampling}

The present study was conducted in the inpatient ward of a tertiary care hospital in Mumbai. Patients, aged more than 65 years of age, having fever of less than 7 days duration who were admitted in our department were included. Those with either respiratory symptoms, gastrointestinal, genitourinary or meningeal symptoms with or without fever were also included in the study. Those suffering from nosocomial infections (infection not present or incubating at the time of admission but occurring within 72 hours of admission in the hospital) were excluded from the study. We included 100 
patients fulfilling the study criteria over a period of 18 months. Patients were explained the purpose of the study and a written consent was obtained before being enrolled in the study. The study was approved by the Institutional Ethics Committee before we enrolled the patients.

\section{Data Collection and Data Analysis}

Demographic data, relevant clinical data and laboratory data was obtained. This included age, sex, occupation, address, addictions, presence or absence of comorbidities such as hypertension, diabetes mellitus, ischemic heart disease, cerebrovascular accident, osteoarthritis, osteoporosis, chronic obstructive airway diseases. Detailed history of every patient was taken in context of the present illness. Each patient was thoroughly examined physically and all the findings were noted. Investigations pertaining to acute infections like complete hemogram with hematocrit, fever profile including peripheral smear for malaria, tests for dengue antibody IgM, dengue NS1 antigen, leptospirosis antibody and widal test, urine routine microscopy and culture sensitivity, stool routine microscopy and culture sensitivity, sputum AFB, gram staining, culture sensitivity were obtained according to patients' signs and symptoms. Other routine investigations like liver function test, renal function test, random blood sugar, serum electrolytes and arterial blood gas were obtained. Cerebrospinal fluid routine microscopy, culture sensitivity and adenosine deaminase levels were obtained in patients with signs and symptoms of meningitis. Imaging studies such as chest x-ray, ultrasonography, CT scan, MRI, 2D Echo were obtained in indicated patients.

Parameters like vitals, serum electrolytes and complete blood count were noted on day 1, 3, 5 and 7 during hospitalization. Outcome was defined as death or discharge or transfer to MICU [Medical intensive care unit] or IRCU [ Intensive Respiratory Care Unit].Acute Physiology, Age, Chronic Health Evaluation II (APACHE II) scoring was applied on the parameters recorded on day 1 and was compared with the outcome. ${ }^{\mathrm{i}}$

Data were compiled and analysed in SPSS (version 23, IBM). The qualitative data were presented as percentages, while the quantitative data were described as means. Association between two quantitative variables was analysed using chi square test or Fischer Exact test and the quantitative parameters at various intervals of time (day 1, day 3, day 5 and day 7]) were analysed using repeated measures ANOVA and pairwise comparison was done using LSD test. $\mathrm{P}$ value of $<0.05$ was considered statistically significant.

\section{Results}

Mean age of the patients was 75.84 years and majority of the patients were in the age group of 66 to 75 years [Table 1]. Two-thirds of the patients were males. On obtaining history of past medical conditions, 60 had comorbid conditions, of which hypertension (32\%) and diabetes mellitus (29\%) were the most common. Other comorbid conditions were ischemic heart disease (15\%), cerebrovascular accident (3\%), hypothyroidism (3\%), bronchial asthma (2\%), chronic myeloid leukemia (1\%), benign prostatic hypertrophy (1\%), dilated cardiomyopathy (1\%), osteoarthritis $(1 \%)$ and osteoporosis (1\%). Addictions of smoking and alcohol were reported by $61 \%$ of the patients.

Pulmonary infections accounted for majority of the infections (43\%) in our study. These include community acquired pneumonia (74.4\%), empyema (2.32\%), acute exacerbations of chronic lung diseases like bronchial asthma, interstitial lung disease and chronic obstructive lung diseases $(23.25 \%)$. Out of them community acquired pneumonia accounts for the majority of pulmonary infections. Out of the total 32 cases of community acquired pneumonia 9 were multi-lobar pneumonia and 23 were lobar pneumonia. Urinary tract infections were the second major cause of acute infection and account for $14 \%$ of total infections. Gastrointestinal infections include acute gastroenteritis and dysentery and they account for $10 \%$ of all the acute infections in our study. Central nervous system infections include pyogenic meningitis, febrile encephalopathy and it accounts for $6 \%$ of all acute infections in our study. Skin infections such as cellulitis account for $4 \%$ of acute infections in our study.Other infections which constitute $23 \%$ of infections include dengue fever, malaria, leptospirosis, liver abscess and constitute second major cause of acute infections. Vitals, total count, serum creatinine, BUN and random blood sugar demonstrated a general decline over a period of one week under treatment [Table 2]. We also observed normalization of serum electrolytes and pO2 levels. During hospitalization, the most common complication was respiratory failure. Other complications have been listed in table 3. Among 17 patients transferred to Medical Intensive Care Unit, 10 died. Overall, 15 patients died during hospital stay. We found APACHE-II score to be significantly higher among patients who expired during hospital stay [Table 4]. Other than that, presence of pulmonary infections were also found to be significantly higher among expired patients.

Table 1: Baseline characteristics of the patients included in the study

\begin{tabular}{|c|c|c|}
\hline Variables & $\mathbf{N}$ & $\%$ \\
\hline \multicolumn{3}{|l|}{ Age distribution } \\
\hline 66 to 75 & 53 & $53 \%$ \\
\hline 76 to 85 & 35 & $35 \%$ \\
\hline$>85$ & 12 & $12 \%$ \\
\hline \multicolumn{3}{|l|}{ Gender distribution } \\
\hline Males & 68 & $68 \%$ \\
\hline Females & 32 & $32 \%$ \\
\hline \multicolumn{3}{|l|}{ Comorbidites $(\mathrm{n}=60)$} \\
\hline Hypertension & 32 & $32 \%$ \\
\hline Diabetes mellitus & 29 & $29 \%$ \\
\hline Ischemic Heart Disease & 15 & $15 \%$ \\
\hline Cerebrovascular accident & 3 & $3 \%$ \\
\hline Hypothyroidism & 3 & $3 \%$ \\
\hline Bronchial asthma & 2 & $2 \%$ \\
\hline Chronic myeloid leukemia & 1 & $1 \%$ \\
\hline Benign prostatic hypertrophy & 1 & $1 \%$ \\
\hline Dilated cardiomyopathy & 1 & $1 \%$ \\
\hline Osteoarthritis & 1 & $1 \%$ \\
\hline Osteoporosis & 1 & $1 \%$ \\
\hline \multicolumn{3}{|l|}{ Addictions } \\
\hline Yes & 61 & $61 \%$ \\
\hline No & 39 & $39 \%$ \\
\hline \multicolumn{3}{|l|}{ Final diagnosis } \\
\hline Pulmonary infections & 43 & $43 \%$ \\
\hline Urinary tract infections & 14 & $14 \%$ \\
\hline Gastrointestinal infections & 10 & $10 \%$ \\
\hline CNS infections & 6 & $6 \%$ \\
\hline Skin infections & 4 & $4 \%$ \\
\hline Others & 23 & $23 \%$ \\
\hline
\end{tabular}

1 
Table 2: Pattern of various patient related parameters in the first 7 days of hospitalization

\begin{tabular}{|c|c|c|c|c|c|}
\hline Parameter & Day 1 & Day 3 & Day 5 & $\begin{array}{l}\text { Day } \\
7\end{array}$ & $\begin{array}{l}\text { p } \\
\text { value* }\end{array}$ \\
\hline $\begin{array}{l}\text { Mean body } \\
\text { temperature }\end{array}$ & 38.31 & 38.26 & 37.84 & 37.4 & $<0.001$ \\
\hline Mean pulse & 106.33 & 105.12 & 97.62 & 89.99 & $<0.001$ \\
\hline Mean respiratory rate & 18.86 & 17.94 & 16.34 & 15.85 & $<0.001$ \\
\hline Mean total count & $\begin{array}{l}13505 . \\
31\end{array}$ & $\begin{array}{l}13305 . \\
51\end{array}$ & $\begin{array}{l}11729 . \\
39\end{array}$ & $\begin{array}{l}1064 \\
6.94\end{array}$ & $<0.001$ \\
\hline $\begin{array}{l}\text { Mean serum } \\
\text { creatinine }\end{array}$ & 1.9 & 1.79 & 1.59 & 1.44 & $<0.001$ \\
\hline Mean BUN & 28.99 & 26.95 & 23.13 & 20.91 & $<0.001$ \\
\hline Mean serum sodium & 134.46 & 133.97 & 135.77 & $\begin{array}{l}136.0 \\
5\end{array}$ & $<0.001$ \\
\hline $\begin{array}{l}\text { Mean serum } \\
\text { potassium }\end{array}$ & 4.17 & 4.12 & 4.09 & 4.05 & $<0.001$ \\
\hline Mean pO2 & 75.9 & 76.97 & 79.41 & 81.81 & $<0.001$ \\
\hline $\begin{array}{l}\text { Mean serum } \\
\text { bicarbonate }\end{array}$ & 21.09 & 21.087 & 22.65 & 22.46 & $<0.002$ \\
\hline $\begin{array}{l}\text { Mean random blood } \\
\text { sugar }\end{array}$ & 151.35 & 130.63 & 118.56 & 112.6 & $<0.001$ \\
\hline
\end{tabular}

\begin{tabular}{l} 
Table 3: Complications and outcome of patients \\
\begin{tabular}{|l|l|l|}
\hline Variables & N & $\%$ \\
\hline Complications & 6 & \\
\hline Septicaemia & 10 & $6 \%$ \\
\hline Respiratory failure & 1 & $10 \%$ \\
\hline Uremic encephalopathy & 2 & $1 \%$ \\
\hline Acute renal failure & 3 & $2 \%$ \\
\hline Multi-organ failure & 7 & $3 \%$ \\
\hline Outcome of Medical Intensive Care Unit transferred patients (n=17) \\
\hline Discharge & 7 & $41 \%$ \\
\hline Death & 10 & $59 \%$ \\
\hline Outcome overall & 85 & $85 \%$ \\
\hline Discharge & 15 & $15 \%$ \\
\hline Death &
\end{tabular} \\
\hline
\end{tabular}

Table 4: Association of patient related variables with the outcome

\begin{tabular}{|l|l|l|l|}
\hline & \multicolumn{2}{l}{ Outcome } & \\
\hline Variables & Discharge & Death & $\mathrm{p}$ value \\
\hline APACHE-2 score & 11.07 & 25.87 & $<0.001^{*}$ \\
\hline Comorbidities & 48 & & \\
\hline Yes & 37 & 12 & 0.08 \\
\hline No & & 3 & \\
\hline Addictions & 50 & & \\
\hline Yes & 35 & 11 & 0.28 \\
\hline No & & 4 & \\
\hline Final diagnosis & 7 & & \\
\hline CNS infections & 10 & 2 & 0.62 \\
\hline GI infections & 33 & 0 & 0.35 \\
\hline Pulmonary infections & 12 & 10 & 0.04 \\
\hline Urinary infections & 4 & 2 & 0.93 \\
\hline Skin infections & *analyzed using student t test; **analyzed using chi-square or Fisher's exact test \\
\hline
\end{tabular}

\section{Discussion}

The present study was conducted to know the pattern of systemic infections among elderly population and various factors associated with their outcomes. Respiratory infections were the most common among our study population. These included community acquired pneumonia, empyema, acute exacerbations of chronic lung diseases like bronchial asthma, interstitial lung disease and chronic obstructive lung diseases. Around $50 \%$ of all community acquired pneumonia occurs in adults older than 65 years of age. ${ }^{[4]}$ Although, a chest X-ray can diagnose pneumonia and its complications, a computed tomograpy may be needed to exclude an underlying malignancy and/or for precise diagnosis of alveolar infiltration. ${ }^{[5]}$ In addition, chronic obstructive pulmonary disease, smoking and alcohol additions predisposes pneumonia caused by Haemophilus influenzae, Moraxella catarrhalis, and Legionella pneumophila. Polymicrobial pneumonia is also frequently encountered in elderly patients admitted to the ICU. ${ }^{[6]}$ Furthermore, presence of pulmonary infections was found to be significantly associated with mortality in our study sample. Similar observations have been made in the past as well. ${ }^{[7]}$

In our patient population, urinary tract infections were the second most common group of diseases. Similar results were demonstrated previously. ${ }^{[8]}$ In the study conducted in South India by Venkatesh et al ${ }^{[9]}, 22 \%$ patients had UTI.While bacteruria is rarely seen in young men, its prevalence reaches $\geq 5 \%$ in those $\geq 65$ years in the community setting. On the other hand, bacteriuria occurs in $5-10 \%$ of women aged $>65$ years as compared to $2-5 \%$ of young women. ${ }^{[5]}$ Previous studies showed that UTI is often erroneously diagnosed with around $40 \%$ of hospitalised elderly admissions due to nonspecific symptoms. UTI was caused due to urinary incontinence, previous history, urogenital surgery and diabetes mellitus. Treating UTI in elderly can also present a challenge to the physician as renal impairment is common in the elderly and often unrecognized. So the calculated glomerulus filtration rate should be determined in all patients and the dosage adjustment of various drugs should be done.

We found poor APACHE-II score to be significantly associated with mortality in our patient population. In a multi-centric cohort study, APACHE-II was also found to be independently associated with mortality among patients aged $\geq 65$ years with sepsis. ${ }^{[10]}$ Palomba et al compared outcomes between elderly ( $\geq 65$ years old) and non-elderly ( $<65$ years old) resuscitated severe sepsis and septic shock patients and determined predictors of death among elderly patients. ${ }^{[1]}$ The authors found that elderly patients had a higher APACHE II score [22 (18-28) versus 19 (15-24); $p<0.001]$, compared to non-elderly patients, although the number of organ dysfunctions did not differ between the groups. APACHE-II score was found to be significant predictor of death among elderly patients. These evidences, in addition to ours, show that APACHE-II assessment of elderly patients admitted with infection should be included as a standard practice.

\section{Conclusion}

Managing infections among elderly patients is a complex issue with high mortality which requires teamwork. A prompt and laborious clinical and diagnostic workup is essential. Empirical, antimicrobial treatment should be initiated as early as possible, covering presumptive offending pathogens in order to prevent mortality. Once a microbiological diagnosis is made, the initial therapy may switch to be targeted and streamlined.A 'multi- disciplinary team' specifically trained to curb acute infections in the geriatric population needs to be created.Prospective studies assessing the long-term impact on functional status and quality of life are necessary. 


\section{References}

1. Sadighi Akha AA. Aging and the immune system: an overview. J Immunol Methods. 2018; 463:21-6.

2. Elias R, Hartshorn K, Rahma O, Lin N, Snyder-Cappione JE. Aging, immune senescence, and immunotherapy: a comprehensive review. Semin Oncol. 2018;45:187-200.

3. Knaus WA, Draper EA, Wagner DP, Zimmerman JE. APACHE II: a severity of disease classification system. Critical care medicine. 1985;13(10):818-29.

4. Blasi F, Akova M, Bonanni P, Dartois N, Sauty E, Webber C, et al. Community- acquired pneumonia in adults: Highlighting missed opportunities for vaccination. Eur J Intern Med. 2017; 37:13-8.

5. Yoshikawa TT, Norman DC. Geriatric infectious diseases: current concepts on diagnosis and management. J Am Geriatr Soc. 2017:65:631-41.

6. Cillóniz C, Ewig S, Ferrer M, Polverino E, Gabarrús A, de la Bellacasa JP, et al. Community-acquired polymicrobial pneumonia in the intensive care unit: aetiology and prognosis. Crit Care. 2011; 15:

\section{R209.}

7. Meyer KC. Impact of aging on the lung. InSeminars in respiratory and critical care medicine. 2010;31(05):519-520

8. Christensen KL, Holman RC, Steiner CA, Sejvar JJ, Stoll BJ, Schonberger LB. Infectious disease hospitalizations in the United States. Clin Infect Dis. 2009; 49:1025-35

9. Venkatesh RK, Prabhu MM, Nandakumar K, RPai KS. Urinary tract infection treatment pattern of elderly patients in a tertiary hospital setup in south India: a prospective study. Journal of Young Pharmacists. 2016;8(2):108.

10. Martin-Loeches I, Guia MC, Vallecoccia MS, Suarez D, Ibarz M, Irazabal M, Ferrer R, Artigas A. Risk factors for mortality in elderly and very elderly critically ill patients with sepsis: a prospective, observational, multicenter cohort study. Annals of intensive care. 2019;9(1):26.

11. Palomba H, Corrêa TD, Silva E, Pardini A, Assuncao MS. Comparative analysis of survival between elderly and non-elderly severe sepsis and septic shock resuscitated patients. Einstein (Sao Paulo). 2015;13(3):357-63.

Copyright: () the author(s), 2019. It is an open-access article distributed under the terms of the Creative Commons Attribution License (CC BY 4.0), which permits authors to retain ownership of the copyright for their content, and allow anyone to download, reuse, reprint, modify, distribute and/or copy the content as long as the original authors and source are cited.

How to cite this article: Patil V, James E, Padnekar S. Etiology and Outcomes in Elderly Patients Admitted For Acute Infections. Acad. J Med. 2019;2(2):155-58.

DOI: dx.doi.org/10.21276/ajm.2019.2.2.40 